\title{
Stability of Thin-Shell Wormholes from Regular ABG Black Hole
}

\author{
M. Sharif *and Saadia Mumtaz ${ }^{\dagger}$ \\ Department of Mathematics, University of the Punjab, \\ Quaid-e-Azam Campus, Lahore-54590, Pakistan.
}

\begin{abstract}
In this paper, we construct thin-shell wormholes from regular AyonBeato and Garcia black hole by employing cut and paste formalism and examine their stability. We analyze attractive and repulsive characteristics of the respective thin-shell wormholes. A general equation of state is assumed as a linear perturbation to explore stability of these constructed wormholes with and without cosmological constant. We consider linear, logarithmic and Chaplygin gas models for exotic matter and evaluate stability regions for different values of charge. It is found that generalized Chaplygin gas model provides maximum stable regions in de Sitter background while modified generalized Chaplygin gas and logarithmic gas yield maximum stable regions in anti-de Sitter spacetime.
\end{abstract}

Keywords: Thin-shell wormholes; Israel formalism; Stability.

PACS: 04.20.Cv; 04.40.Nr; 04.40.Gz; 04.70.Bw.

\section{Introduction}

The stability of wormholes under perturbations has been a challenging issue for physicists in general relativity. A "wormhole" is like a hole with two ends

*msharif.math@pu.edu.pk

†sadiamumtaz17@gmail.com 
associating distant regions of the universe which takes much less time for traveling as compared to normal travel time. Besides the lack of observational evidence, wormholes are considered to be interconvertible with black holes (BH) [1]. The Einstein-Rosen bridge is an example of non-traversable wormhole in which wormhole throat shrinks leading to the existence of event horizon [2]. Traversable wormholes have no event horizon which allow the two way observer's motion without any hindrance [3].

The existence of exotic matter at the wormhole throat gives rise to the violation of null (NEC) and weak energy conditions (WEC) which is an important ingredient for traversable wormholes. The weakest one is NEC whose violation leads to the violation of WEC and strong energy conditions. Israel thin-shell formalism is the most useful technique to compute presuure and energy density which idetifies exotic matter at thin-shell [4]. Thin-shell wormholes belong to one of the wormhole classes in which this matter is restricted at the shell. The unavoidable amount of exotic matter is a debatable issue for physical viability of thin-shell wormholes. This amount can be quantified by the volume integral theorem for shell which is consistent with the concept that a small quantity of exotic matter is required to support wormhole [5]. Visser [6] proposed cut and paste procedure for polyhedral wormholes which could make infinitesimally small contribution of exotic matter such that a traveler encountering the wormhole does not feel any tidal force.

Thin-shell wormholes can be constructed from the family of regular BHs which are static spherically symmetric and asymptotically flat regions with regular (singularity-free) centers. The horizons are indeed formed, while singularities are avoided in the interior of BHs. We can choose regular BHs for wormhole construction due to their viability in high energy collisions. Bardeen was the first, who proposed a regular BH solution with specific mass to charge ratio [7]. Ayon-Beato and Garcia [8] found another regular BH coupled with nonlinear electrodynamics known as Ayon-Beato and Garcia (ABG) BH. Hayward [9] proposed a similar type of regular BH which can be reduced to de Sitter and Schwarzschild spacetimes in the limit $r \rightarrow 0$ and $r \rightarrow \infty$, respectively. Recently, Halilsoy et al. [10] studied stability of regular Hayward wormhole configurations.

It is well-known that the implementation of linear perturbations or equation of state (EoS) has remarkable significance in the investigation of wormhole stability. In this context, Kim and Lee [11] analyzed the role of charge on stability of Reissner-Nordström wormholes. Sharif and collaborators [12][14] explored the role of electric charge on the stability of spherical as well as 
cylindrical thin-shell wormholes. Lobo et al. [15] introduced a novel approach to study wormhole stability under linearized perturbations and analyzed that matter content supporting wormhole throat minimally violates NEC.

It has been a burning issue for cosmologists to have some physically suitable models for exotic matter. Different candidates of dark energy have been proposed in this regard like phantom energy [16, quintessence [17] and family of Chaplygin gas (CG) [18]. Eiroa [19] assumed generalized Chaplygin gas (GCG) to study the dynamics of spherical thin-shell wormholes. Sharif and Azam [20] discussed stability of Reissner-Nordström wormhole configurations by taking modified generalized Chaplygin gas (MGCG) and found both stable as well as unstable configurations. The stability of spherically symmetric wormholes have been studied in the context of two different CG models [21, 22]. Mazharimousavi and Halilsoy [23] analyzed the role of angular momentum on the stability of counter-rotating wormhole solutions in the vicinity of linear gas EoS.

This paper investigates stability of regular ABG thin-shell wormholes by considering different dark energy models. The paper is organized in the following format. In section $\mathbf{2}$, we construct regular ABG wormhole solutions and discuss various physical aspects. The standard approach for stability analysis is given in section $\mathbf{3}$. Section $\mathbf{4}$ deals with stability formalism of the regular ABG thin-shell wormholes in the context of linear, logarithmic and CG models. We assume small velocity dependent perturbations to investigate wormhole stability in section 5. Finally, we provide a brief overview of the obtained results in the last section.

\section{Regular ABG Thin-Shell Wormhole}

The study on global regularity of BHs has attracted the attention of many researchers. None of the regular BHs are exact solutions to the field equations without any physically reasonable source. To derive the nonlinear electromagnetic field, one requires to enlarge the class of electrodynamics to nonlinear ones [8]. These regular BHs behave as ordinary Reissner-Nordström BH solutions and the existence of these solutions does not contradict the singularity theorems [24]. This motivates us to discuss stability of viable wormhole solutions coupled with nonlinear electrodynamics. The static spherically symmetric nonsingular ABG $\mathrm{BH}$ is given by

$$
d s^{2}=-G(r) d t^{2}+G^{-1}(r) d r^{2}+r^{2}\left(d \theta^{2}+\sin ^{2} \theta d \phi^{2}\right),
$$


where

$$
G(r)=1-\frac{2 M r^{2}}{\left(r^{2}+Q^{2}\right)^{\frac{3}{2}}}+\frac{r^{2} Q^{2}}{\left(r^{2}+Q^{2}\right)^{2}},
$$

$M$ is the mass and $Q$ denotes the charge. We choose this regular $\mathrm{BH}$ for wormhole construction because a regular system can be constructed from a finite energy and its evolution is more acceptable. The corresponding spacetime becomes regular at $r=0$ and behaves as Reissner-Nordström BH for $r \rightarrow \infty$.

Its event horizon is the largest root of the equation

$$
r^{4}+Q^{4}+3 r^{2} Q^{2}-2 M r^{2} \sqrt{r^{2}+Q^{2}}=0 .
$$

If real and positive solutions of Eq.(3) exist, the spacetime yields a BH while if there is no such solution then spacetime is fully regular without event horizon [25]. The analysis of the roots shows that the above expression constitutes positive real roots if $0<Q<2 M$. The critical value of charge $Q_{c r i t}=$ $0.634 M$ separates the $\mathrm{BH}$ and the no-horizon ABG spacetime. In no-horizon case, the $\mathrm{ABG}$ spacetime is regular at all radii $r \geq 0$. For $Q \geq Q_{\text {crit }}$, there are no horizons while the given spacetime admits two horizons for $Q<Q_{\text {crit }}$ leading to a non-extremal BH. The Schwarzschild $\mathrm{BH}$ is recovered for $Q=0$. For no-horizon solutions, ABG spacetime allows circular geodesic motion of test particles and photons. There exist two photon circular geodesics in the no-horizon spacetime in which the outer one is unstable relative to radial perturbations and the inner one is stable [25, 26]. We also consider ABG regular $\mathrm{BH}$ coupled with a cosmological constant $(\Lambda)$ in the metric function as 27

$$
G(r)=1-\frac{2 M r^{2}}{\left(r^{2}+Q^{2}\right)^{\frac{3}{2}}}+\frac{r^{2} Q^{2}}{\left(r^{2}+Q^{2}\right)^{2}}-\frac{\Lambda r^{2}}{3},
$$

which reduces to Eq.(2) as $\Lambda \rightarrow 0$. This metric describes a regular ABG $\mathrm{BH}$ in de Sitter $(\Lambda>0)$ and anti-de Sitter $(\Lambda<0)$ spacetimes. The static radius of the given spacetime plays an important role for the location of its horizons and no-horizon solutions [28]-30]. In the case of de Sitter and antide Sitter regular ABG spacetimes, the metric function $G(r)$ can have at most three distinct roots leading to three horizons in which $r_{c}$ is the cosmological horizon, $r_{h}$ is the event horizon and $r_{b}$ is the inner horizon [31.

For the construction of a timelike thin-shell wormhole, we apply standard cut and paste method such that the resulting 4D copies are glued at the hypersurface $\Sigma^{ \pm}=\Sigma=\{r=a\}$. The wormhole throat should fulfill the 
flare-out condition for which exotic matter is required. To avoid singularities and unification of event and cosmological horizons, we must have $r_{h}<a_{0}<r_{c}$ for the possibility of static wormhole solutions from de Sitter and anti-de Sitter spacetimes. The existence of horizons in wormhole configuration do not matter because the wormhole throat must be taken out of horizon, i.e., $a_{0}>r_{h}$ for traversability of thin-shell wormholes [32]. We consider a 3D timelike spacetime at the shell defined by

$$
d s^{2}=-d \tau^{2}+a^{2}(\tau)\left(d \theta^{2}+\sin ^{2} \theta d \phi^{2}\right)
$$

where $\tau$ is the proper time on the shell. The unit four-vector normals $n_{\alpha}^{ \pm}$to $\mathcal{M}^{ \pm}$are defined by

$$
n_{\alpha}^{ \pm}= \pm\left|g^{\mu \nu} \frac{\partial \eta}{\partial x^{\mu}} \frac{\partial \eta}{\partial x^{\nu}}\right|^{-\frac{1}{2}} \frac{\partial \eta}{\partial x^{\alpha}}=\left(-\dot{a}, \frac{\sqrt{G(r)+\dot{a}^{2}}}{G(r)}, 0,0\right)
$$

satisfying the relation $n^{\alpha} n_{\alpha}=\epsilon=1$. We implement Israel formalism for the dynamical evolution of thin-shell which allows matching of two spacetime regions separated by $\Sigma$. We use Lanczos equations to determine surface stresses at wormhole throat given by

$$
S_{j}^{i}=\frac{1}{8 \pi}\left\{[K] \delta_{j}^{i}-\left[K_{j}^{i}\right]\right\}
$$

where $\left[K_{j}^{i}\right]$ represents the extrinsic curvature tensor and $K=\operatorname{tr}\left[K_{j}^{i}\right]$. The surface stresses at the shell are determined as [12]

$$
\begin{aligned}
\sigma & =-\frac{1}{2 \pi a} \sqrt{G(a)+\dot{a}^{2}} \\
p & =\frac{1}{4 \pi}\left[\frac{\sqrt{G(a)+\dot{a}^{2}}}{a}+\frac{2 \ddot{a}+G^{\prime}(a)}{2 \sqrt{G(a)+\dot{a}^{2}}}\right] .
\end{aligned}
$$

For the existence of any physically realistic matter, we need to check the energy conditions. The sum of surface stresses of matter indicates violation of NEC leading to the presence of exotic matter. The amount of this matter should be minimized for the sake of viable wormhole configurations. We see from Figure 1 that $\sigma<0$ and $\sigma+p<0$ showing the violation of NEC and WEC for different values of $Q$ and $a$ (with and without $\Lambda$ ). 

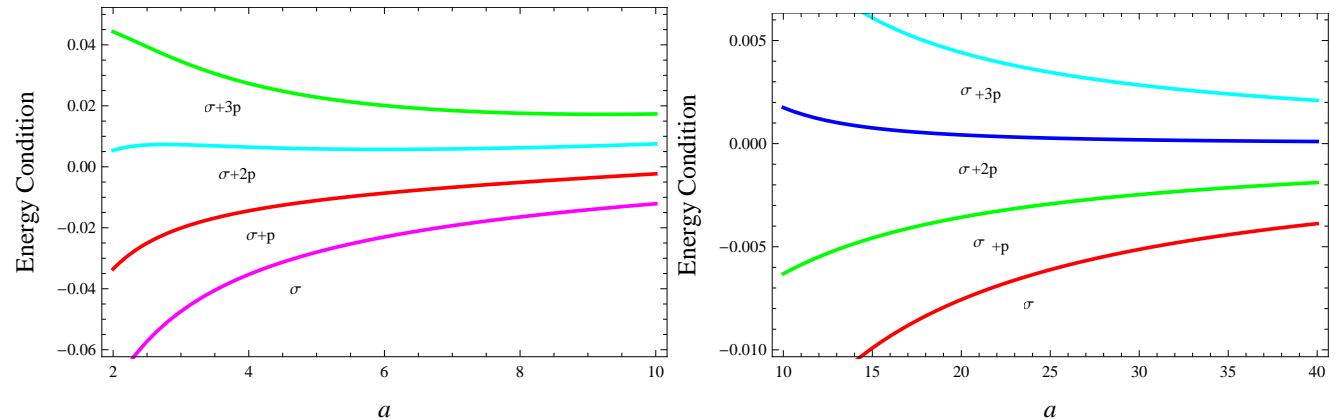

Figure 1: Plots of energy conditions with $\Lambda=0.1$ (left hand side) and without $\Lambda$ (right hand side) corresponding to $\frac{Q}{M}=1.1$.

Now we analyze attractive and repulsive behavior [34] of the constructed wormhole solutions for which observer's four-acceleration is given by

$$
a^{\mu}=u_{; \nu}^{\mu} u^{\nu}
$$

where $u^{\mu}=\frac{d x^{\mu}}{d \tau}=\left(\frac{1}{\sqrt{G(r)}}, 0,0,0\right)$ is the observer's four-velocity. The non-zero four-acceleration component is computed as

$a^{r}=\Gamma_{t t}^{r}\left(\frac{d t}{d \tau}\right)^{2}=\frac{3 M r^{3}}{\left(r^{2}+Q^{2}\right)^{\frac{5}{2}}}-\frac{2 M r}{\left(r^{2}+Q^{2}\right)^{\frac{3}{2}}}+\frac{r Q^{2}}{\left(r^{2}+Q^{2}\right)^{2}}-\frac{2 r^{3} Q^{2}}{\left(r^{2}+Q^{2}\right)^{3}}-\frac{\Lambda r}{3}$,

and the geodesic equation has the following form

$$
\frac{d^{2} r}{d \tau^{2}}=-\Gamma_{t t}^{r}\left(\frac{d t}{d \tau}\right)^{2}=-a^{r}
$$

A wormhole will be attractive in nature if $a^{r}>0$ while it will exhibit repulsive characteristics for $a^{r}<0$. The attractive and repulsive characteristics of the regular ABG thin-shell wormholes are analyzed corresponding to different values of charge. Figure 2 shows respective plots with and without $\Lambda$.

It is well-known fact that exotic matter supports the wormhole throat to make it traversable. The total amount of this matter at shell is quantified by the integral theorem [5]

$$
\Omega=\int_{0}^{2 \pi} \int_{-\infty}^{+\infty}\left[\rho+p_{r}\right] \sqrt{-g} d R d \phi
$$



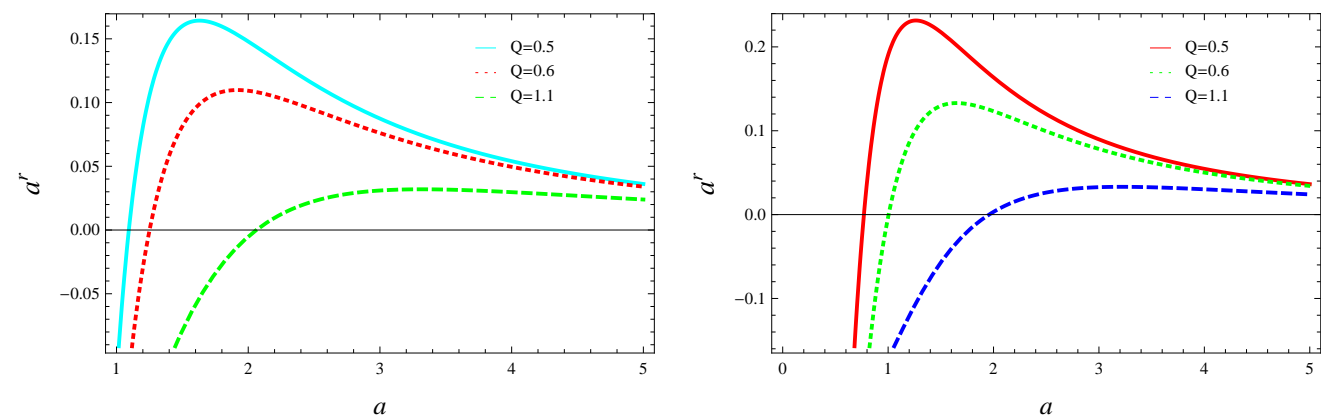

Figure 2: Plots of $a^{r}$ with $\Lambda=0.1$ (left hand side) and without $\Lambda$ (right hand side) for different values of $\frac{Q}{M}$. The wormhole will be attractive or repulsive corresponding to $a^{r}>0$ or $a^{r}<0$, respectively.

The wormhole shell, being thin, does not apply any pressure $\left(p_{r}=0\right)$. Taking $\rho=\delta(R) \sigma(a)$, we have

$$
\Omega_{a}=\left.\int_{0}^{2 \pi}[\rho \sqrt{-g}]\right|_{r=a} d \phi=2 \pi a \sigma(a)
$$

This expression through surface energy density $\sigma\left(a_{0}\right)$ becomes

$$
\Omega_{a}=-\frac{\left(a_{0}^{4}+3 a_{0}^{2} Q^{2}-2 M a_{0}^{2} \sqrt{a_{0}^{2}+Q^{2}}+Q^{4}-\frac{\Lambda a_{0}^{2}}{3}\left(a_{0}^{2}+Q^{2}\right)^{2}\right)^{\frac{1}{2}}}{a_{0}^{2}+Q^{2}}
$$

where $a_{0}$ represents static throat radius of ABG thin-shell wormhole. The behavior of exotic matter is given in Figure $\mathbf{3}$ which shows how the amount of exotic matter varies by varying charge.

\section{Conditions for Wormhole Stability}

In this section, we provide a standard approach for the wormhole stability under linear perturbations. The surface stresses corresponding to static wormhole solution $\left(a=a_{0}\right)$ become

$$
\sigma_{0}=-\frac{\sqrt{G\left(a_{0}\right)}}{2 \pi a_{0}}, \quad p_{0}=\frac{1}{4 \pi}\left[\frac{\sqrt{G\left(a_{0}\right)+}}{a_{0}}+\frac{G^{\prime}\left(a_{0}\right)}{2 \sqrt{G\left(a_{0}\right)}}\right] .
$$



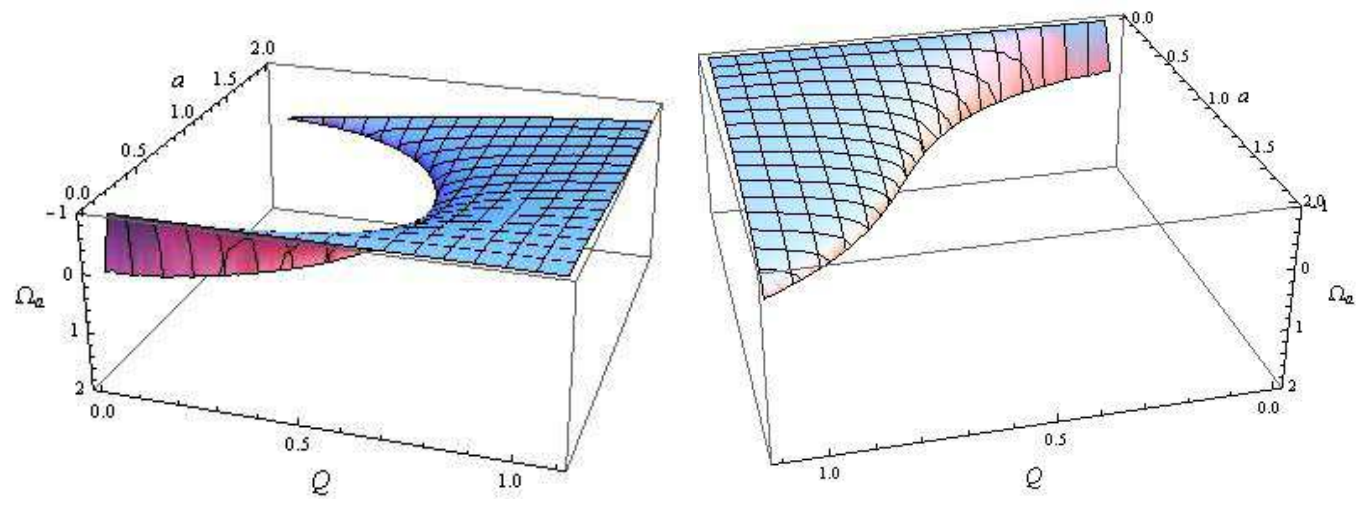

Figure 3: Plots for the total amount of exotic matter with $\Lambda=0.1$ (left hand side) and without $\Lambda$ (right hand side) with different values of charge.

In order to explore wormhole stability, we consider barotropic EoS as a linear perturbation in the form

$$
p=\Phi(\sigma),
$$

where $\Phi(\sigma)$ is chosen arbitrarily. This covers the polytropic EoS $p \approx \sigma^{1+\frac{1}{n}}$ with $0 \leq n<\infty$. The energy density and pressure follow the conservation identity $S_{; j}^{i j}=0$ which, for the induced metric (5), becomes

$$
\frac{d}{d \tau}(\sigma \Psi)+p \frac{d \Psi}{d \tau}=0
$$

where $\Psi=4 \pi a^{2}$ is the area of wormhole throat. The equation of motion for thin-shell can be obtained by setting Eq.(8) as $\dot{a}^{2}+\Delta(a)=0$, which describes wormhole dynamics whereas $\Delta(a)$ is the potential function defined by

$$
\Delta(a)=G(a)-[2 \pi a \sigma]^{2} .
$$

The stability of wormhole static solution requires $\Delta^{\prime}\left(a_{0}\right)=0=\Delta\left(a_{0}\right)$ and $\Delta^{\prime \prime}\left(a_{0}\right)>0$. In this context, we substitute Eq.(15) and $\sigma^{\prime}=\frac{\dot{\sigma}}{\dot{a}}$ in conservation equation which yields

$$
\sigma^{\prime}=-\frac{2}{a}(\sigma+\Phi)
$$

leading to

$$
\sigma^{\prime \prime}=\frac{2}{a^{2}}(\sigma+\Phi)\left(3-a \Phi^{\prime}\right)
$$


The first derivative of Eq.(17) through (18) takes the form

$$
\Delta^{\prime}\left(a_{0}\right)=G^{\prime}\left(a_{0}\right)+8 \pi^{2} a_{0} \sigma_{0}\left[\sigma_{0}+2 p\left(\sigma_{0}\right)\right]
$$

which further turns out to be

$$
\Delta^{\prime \prime}\left(a_{0}\right)=G^{\prime \prime}\left(a_{0}\right)-8 \pi^{2}\left\{\left[\sigma_{0}+2 p_{0}\right]^{2}+2 \sigma_{0}\left[\sigma_{0}+p_{0}\right]\left[1+2 \Phi^{\prime}\left(\sigma_{0}\right)\right]\right\},
$$

where $\Phi_{0}=p_{0}$.

\section{Models for Exotic Matter}

Here we study stability of regular ABG thin-shell wormholes in the context of different dark energy models. The choice of model has remarkable significance in the dynamical investigation of thin-shell wormholes. Recently, the dynamics of regular wormhole solutions has been examined by taking linear, logarithmic and Chaplygin gas models [10, 33]. In this paper, we consider these fluids to study stability of regular ABG wormholes. This enables us to examine the effect of electric charge and other parameters on the wormhole stability. In the following, we discuss stability analysis in the context of above mentioned candidates for exotic matter.

\subsection{Linear Gas}

We consider a linear gas [34] defined by an EoS

$$
\Phi=p_{0}+\mu\left(\sigma-\sigma_{0}\right)
$$

here $\mu$ represents a constant parameter. The first derivative of this equation w.r.t. $\sigma$ leads to $\Phi^{\prime}\left(\sigma_{0}\right)=\mu$. It is found that $\Delta(a)$ and $\Delta^{\prime}(a)$ vanish by substituting $\sigma\left(a_{0}\right)$ and $p\left(a_{0}\right)$. We explore the possibility for the existence of stable wormhole solutions with increasing values of electric charge. Figure 4 shows stable solutions (red curves) of the regular ABG wormholes with

$\frac{Q}{M}=0,0.634,0.77,1.1$. Here $\frac{Q}{M}=0$ represents the Schwarzschild case. We also plot the metric function $G(r)$ to evaluate the position of wormhole throat and event horizon of ABG BH, where we choose $a_{0}=r$ at thin-shell (hypersurface $\Sigma$ ). It is mentioned here that we choose the same range for both $a_{0}$ and $r$-values to plot the metric function $G(r)$. To avoid the existence of event horizon in the wormhole configuration, we take $a>r_{h}$ for traversable 
thin-shell wormholes. We analyze more stable solutions for positive as well as negative values of parameter $\mu$ with $\frac{Q}{M}=0.634$. It is observed that the stability region decreases by increasing the values of $\frac{Q}{M}$.

We also analyze the stability of regular ABG wormhole configurations in de Sitter $(\Lambda=0.1)$ and anti-de Sitter $(\Lambda=-0.5)$ backgrounds. The wormhole throat must satisfy $r_{h}<a_{0}<r_{c}$ for the viability of wormhole solutions. The respective results show more stable wormholes for de Sitter case as compared to anti-de Sitter spacetime (Figure 5). This supports the fact that effects of linear gas become more significant for the regions of stability in de Sitter background.

\subsection{Chaplygin Gas}

Now we assume CG governed by an EoS of the form

$$
\Phi(\sigma)=p_{0}+\mu\left(\frac{1}{\sigma}-\frac{1}{\sigma_{0}}\right),
$$

where $\Phi^{\prime}\left(\sigma_{0}\right)=-\frac{\mu}{\sigma_{0}^{2}}$. The results in Figures 6 correspond to CG with different values of charge. We also plot the graphical results for de Sitter and anti-de Sitter spacetimes as shown in Figure 7. In all these cases, only one stable solution is investigated for different values of $\frac{Q}{M}$. We find one stable region for $\frac{Q}{M}=0.77,1.1$ with negative values of $\mu$. It is found that CG gives minimum stable regions for the respective wormhole solutions as compared to the other EoS for exotic matter. We also analyze that negative values of $\mu$ provide one stable solution in de Sitter and anti-de Sitter cases.

\subsection{Generalized Chaplygin Gas}

Here we take GCG model for which EoS is given by

$$
\Phi(\sigma)=p_{0}+\mu\left(\frac{1}{\sigma^{\gamma}}-\frac{1}{\sigma_{0}^{\gamma}}\right)
$$

where $\gamma$ is EoS parameter. We are interested to check its effect on the wormhole stability. For this purpose, we choose $\mu=p_{0} \sigma^{\gamma}$ which makes the above EoS in the form

$$
\Phi(\sigma)=p_{0}\left(\frac{\sigma_{0}}{\sigma}\right)^{\gamma}
$$



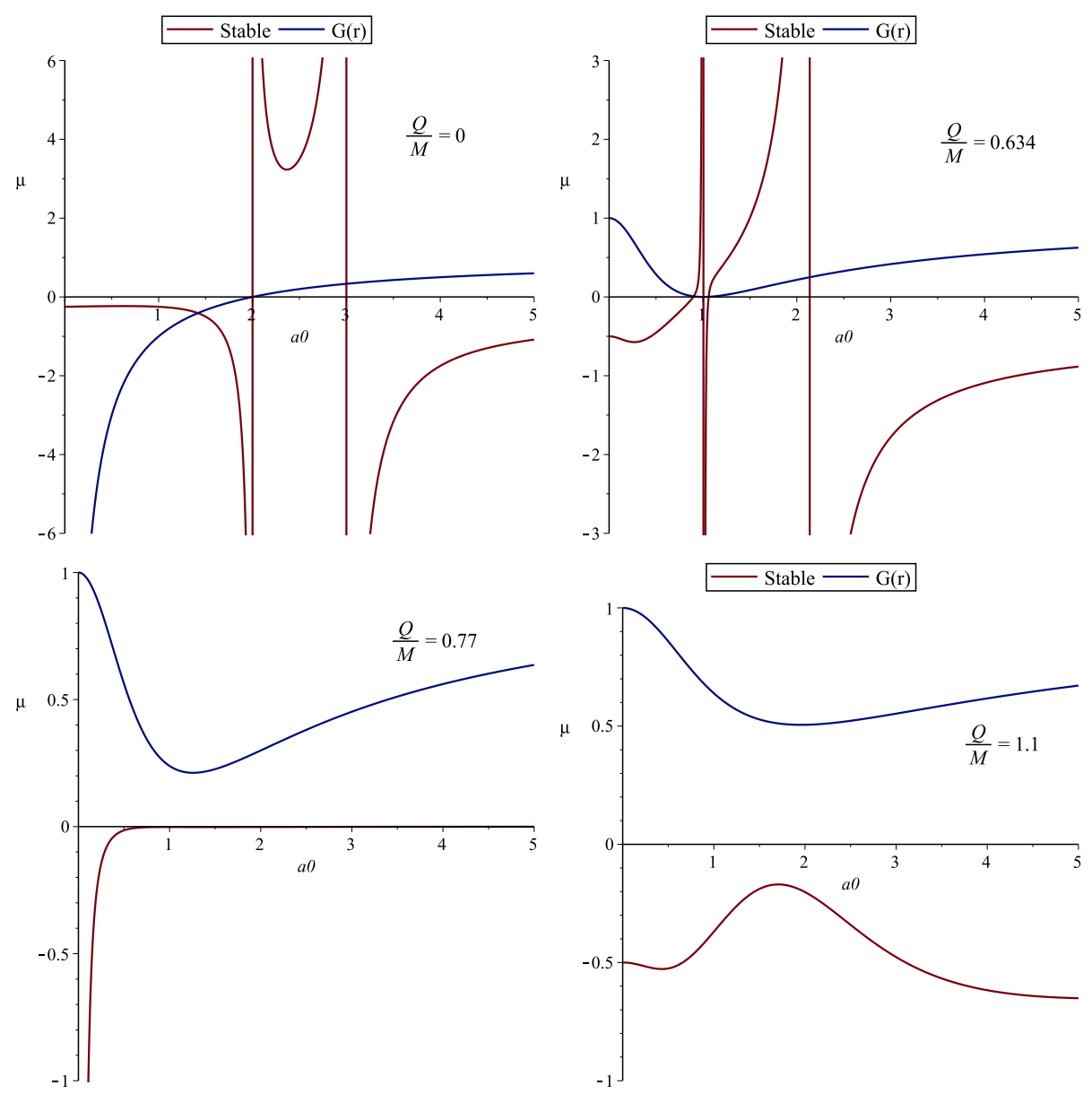

Figure 4: Plots for regular ABG wormholes by taking linear gas EoS and $\frac{Q}{M}=0,0.634,0.77,1.1$. The red and blue curves corresponds to stable regions and the metric function, respectively. We plot throat radius $a_{0}$ and parameter $\mu$ along abscissa and ordinate, respectively, where $a_{0}=r$ at thinshell. 

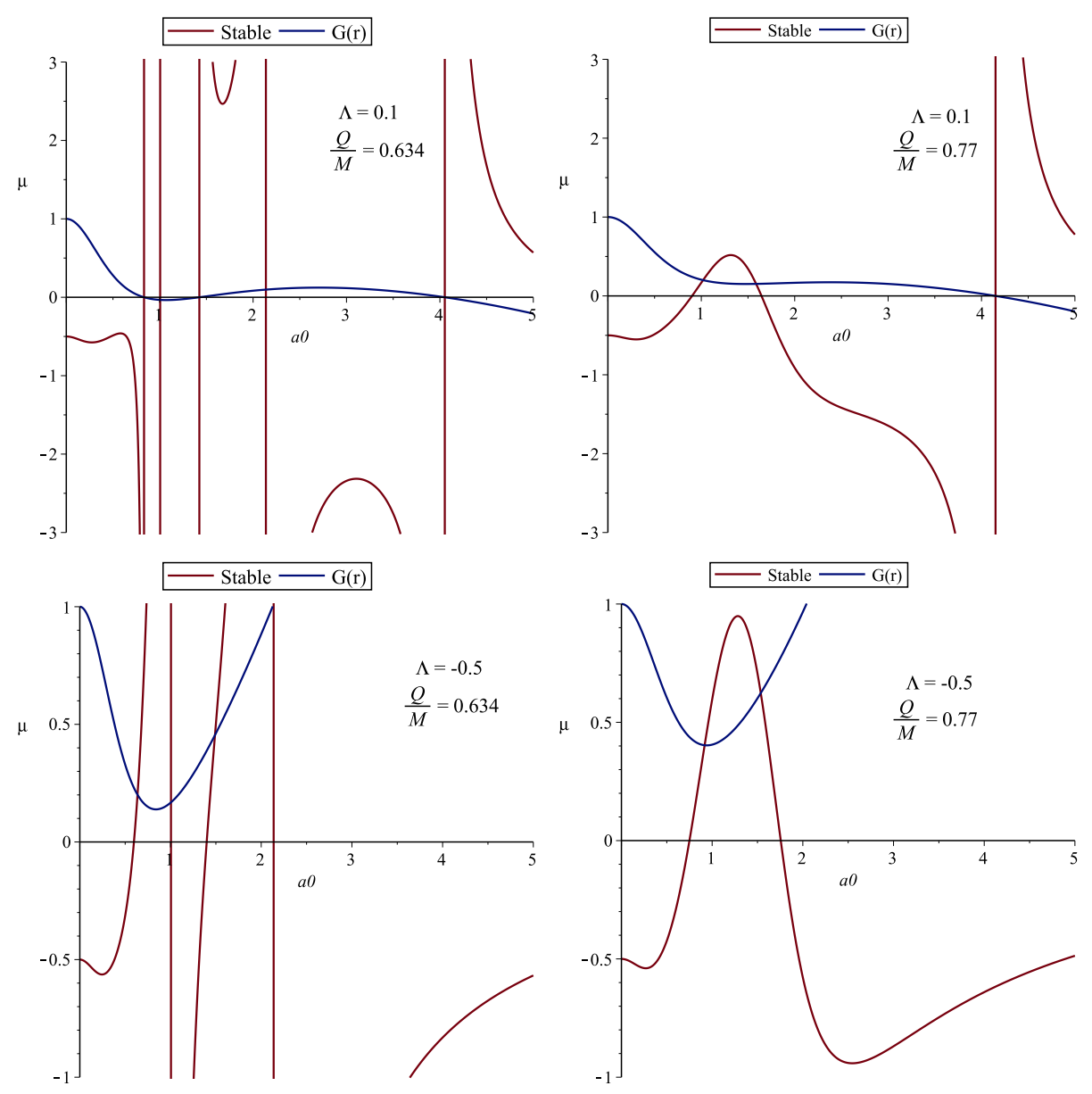

Figure 5: Plots of stable regular ABG wormholes with linear gas in de Sitter $(\Lambda=0.1)$ and anti-de Sitter $(\Lambda=-0.5)$ backgrounds. 

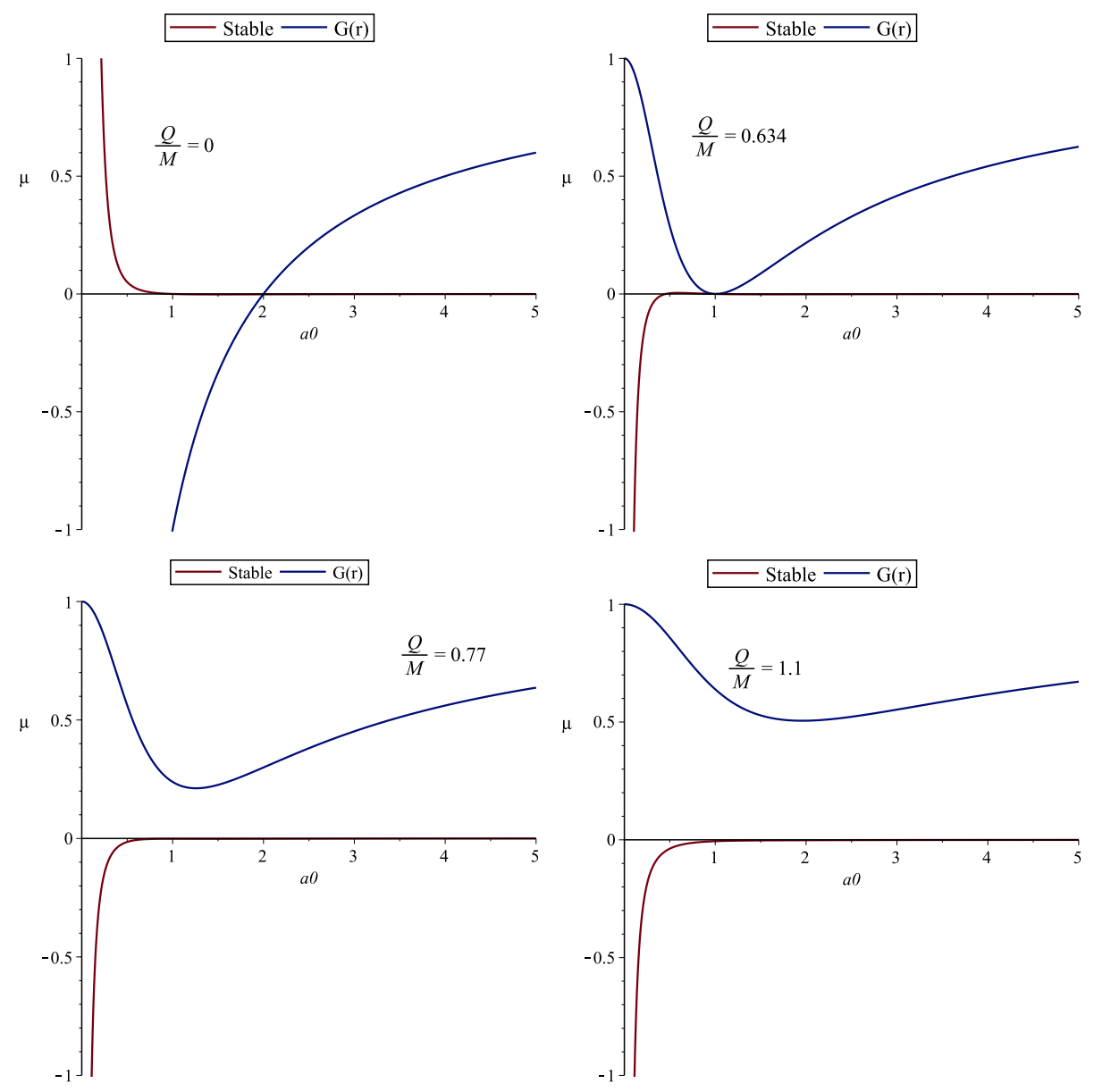

Figure 6: Plots for stable ABG wormhole solutions with CG EoS and $\frac{Q}{M}=$ $0,0.634,0.77,1.1$. We plot throat radius $a_{0}$ and parameter $\mu$ along abscissa and ordinate, respectively, where $a_{0}=r$ at thin-shell. 

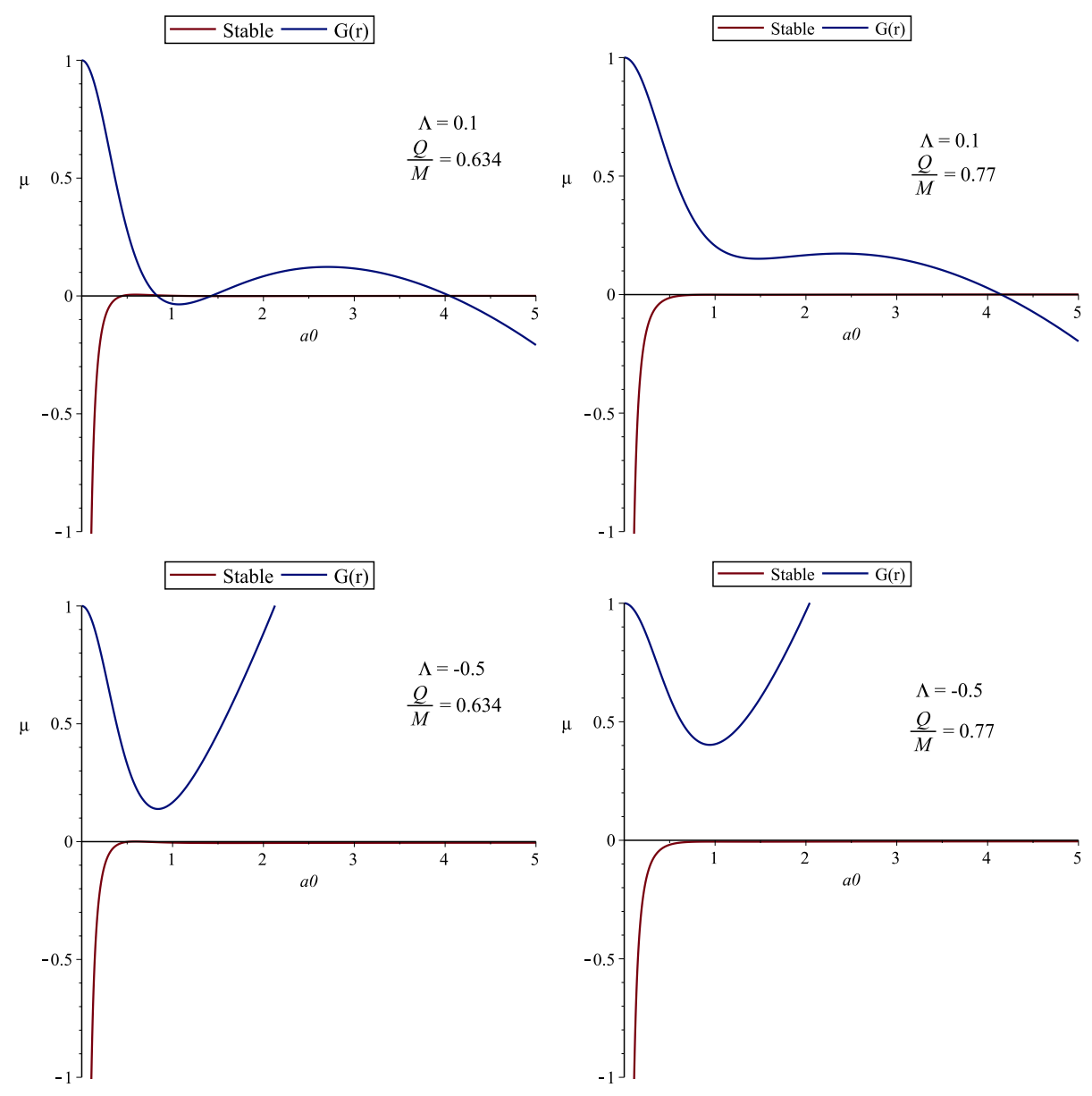

Figure 7: Plots for stable ABG wormholes with CG EoS, $\Lambda=0.1,-0.5$ and $\frac{Q}{M}=0.634,0.77$. 

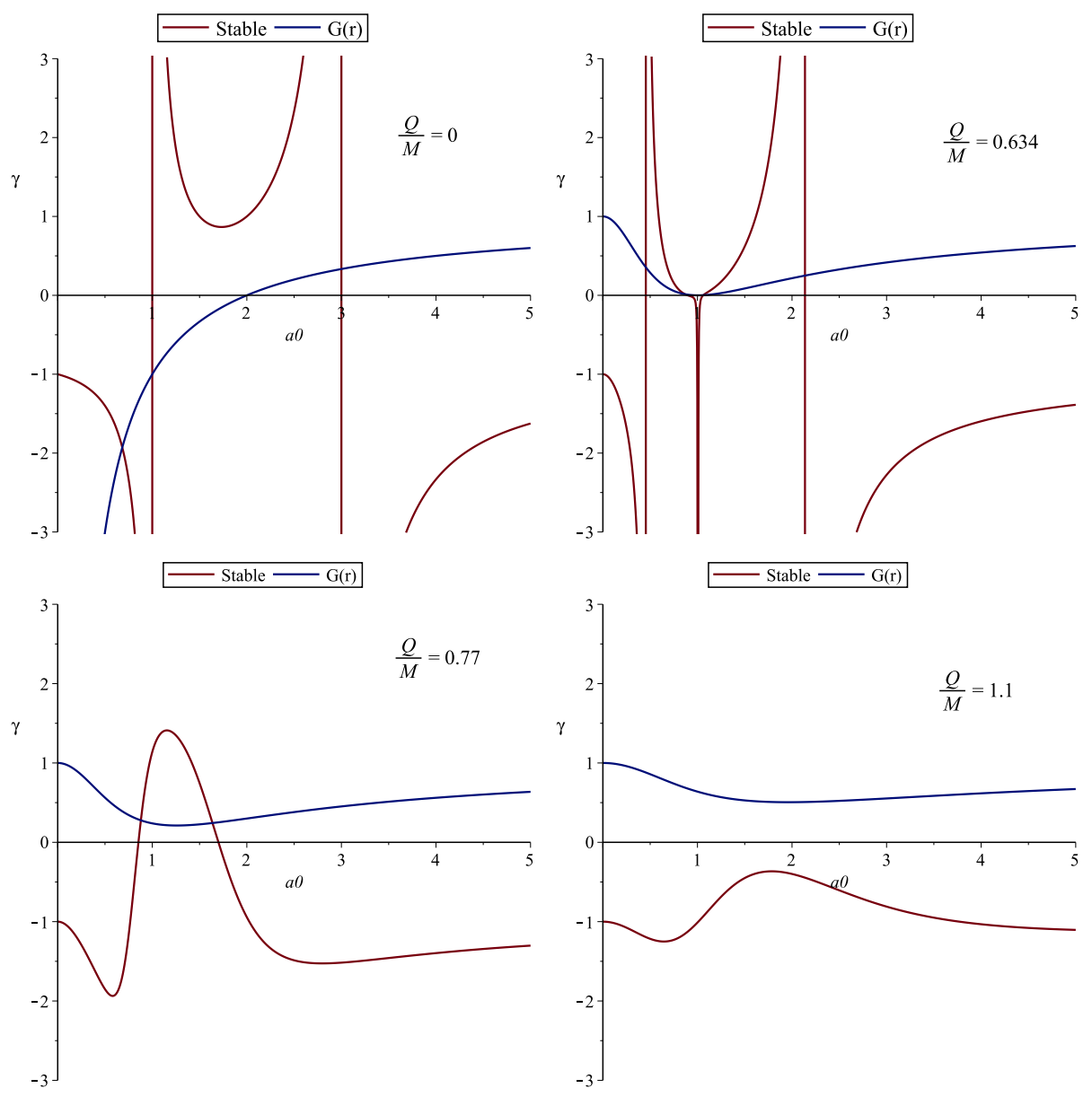

Figure 8: Plots for stable regular ABG wormholes with GCG and $\frac{Q}{M}=$ $0,0.634,0.77,1.1$. We plot throat radius $a_{0}$ and parameter $\gamma$ along abscissa and ordinate, respectively, where $a_{0}=r$ at thin-shell. 

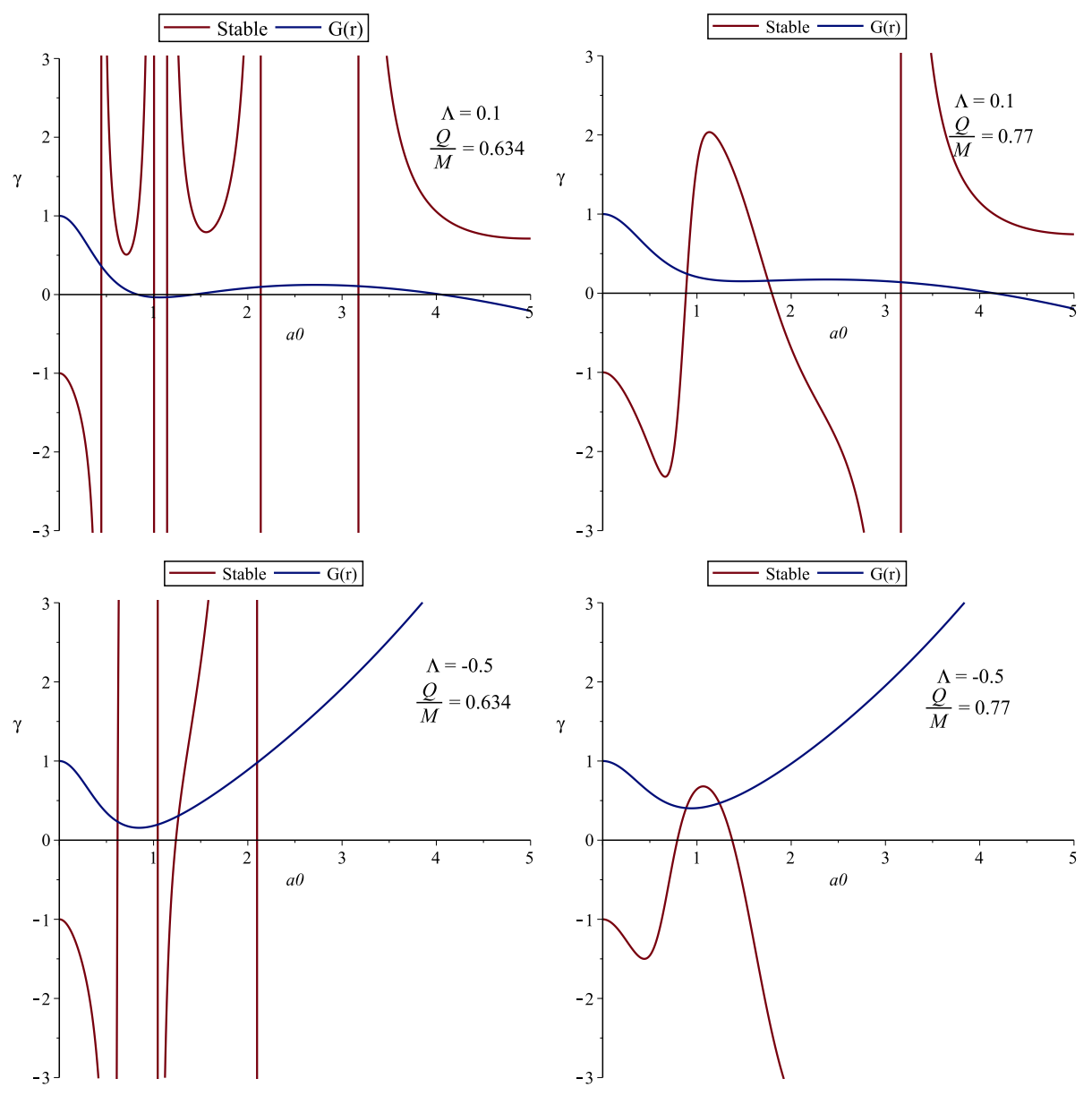

Figure 9: Plots for stability of regular ABG wormhole solutions with GCG and $\Lambda=0.1,-0.5$. 
such that $\Phi^{\prime}\left(\sigma_{0}\right)=-\frac{p_{0}}{\sigma_{0}} \gamma$. We plot the respective stability regions numerically as shown in Figure 8. For GCG, we analyze maximum stability areas for $\frac{Q}{M}=0.634$ which are decreased by increasing $\frac{Q}{M}$ such that only one stable region is obtained for $\frac{Q}{M}=1.1$. We also plot results for the stability of the respective wormholes for $\Lambda=0.1,-0.5$ as shown in Figure 9. These results show that more stable regions exist as compared to the case without $\Lambda$. It is noted that GCG has remarkable importance to provide maximum stability regions for de Sitter ABG wormholes.

\subsection{Modified Generalized Chaplygin Gas}

It is the well-known modification of GCG defined by an EoS

$$
\Phi(\sigma)=p_{0}+\xi_{0}\left(\sigma-\sigma_{0}\right)-w\left(\frac{1}{\sigma^{\gamma}}-\frac{1}{\sigma_{0}^{\gamma}}\right),
$$

where $\xi_{0}$ is a free parameter. Differentiating it w.r.t $\sigma$, we have

$$
\Phi^{\prime}\left(\sigma_{0}\right)=\xi_{0}+\frac{w \gamma}{\sigma_{0}^{\gamma+1}} .
$$

We plot the corresponding graphs in Figure $\mathbf{1 0}$ for $\xi_{0}=\gamma=1$ and different values of charge. For MGCG, we find the possibility of more stability regions which decreases gradually by increasing $\frac{Q}{M}$ and reduces to one stable region for $\frac{Q}{M}=1.1$. For $\Lambda=0.1,-0.5$, we find that the increasing value of $\frac{Q}{M}$ decreases the possibility of stable solutions (Figure 11). Here we analyze maximum stable regions for $\Lambda=-0.5$ and $\frac{Q}{M}=0.634$ which supports the fact that more stable regions exist in anti-de Sitter case as compared to de Sitter case.

\subsection{Logarithmic Gas}

Now we consider logarithmic gas governed by the following EoS

$$
\Phi(\sigma)=p_{0}+w \ln \left|\frac{\sigma}{\sigma_{0}}\right|
$$

where $\Phi^{\prime}\left(\sigma_{0}\right)=\frac{w}{\sigma_{0}}$. The corresponding stable ABG thin-shell wormholes for $\frac{Q}{M}=0,0.634,0.77,1.1$ without $\Lambda$ are shown in Figure 12. It is found 

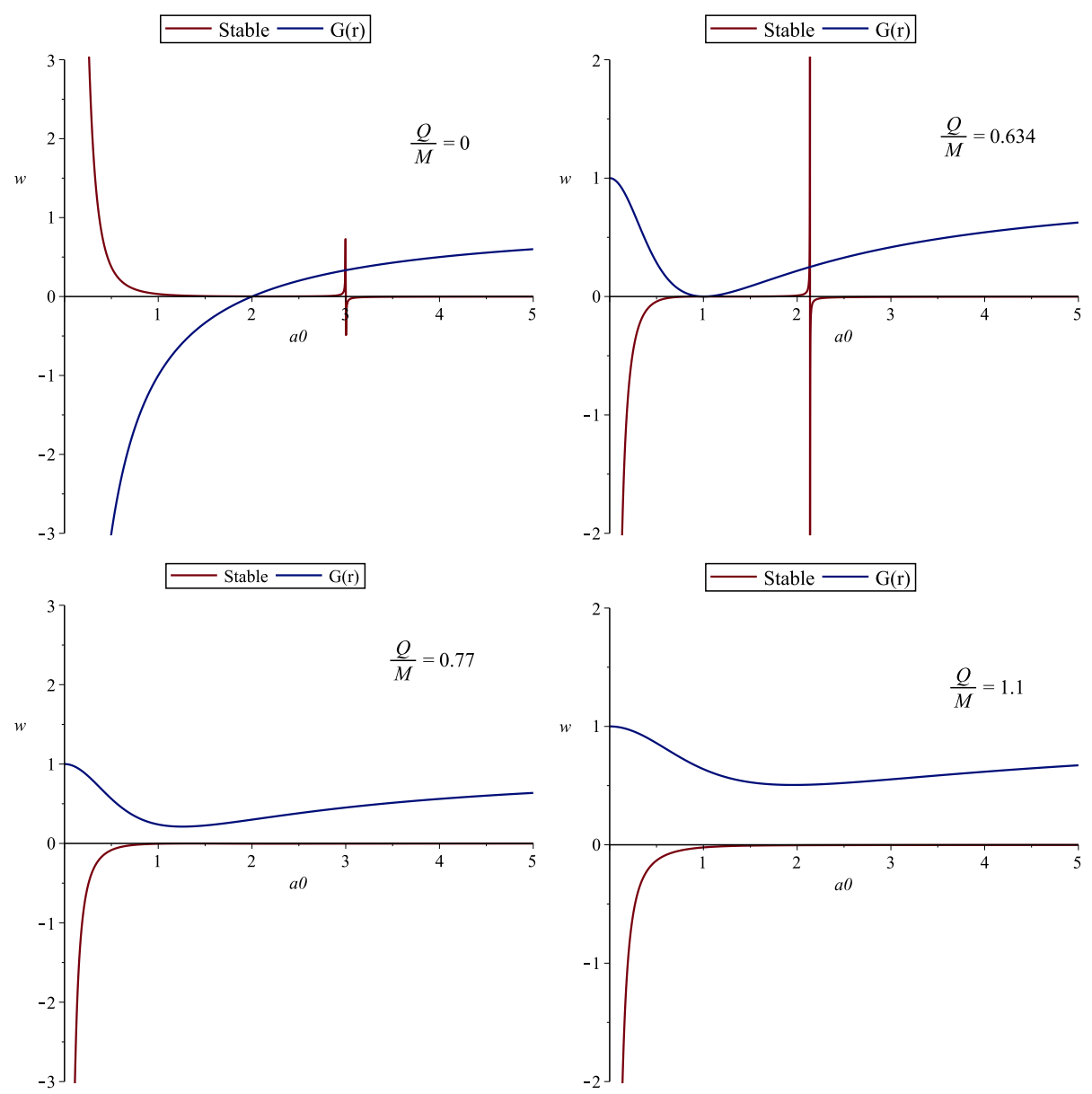

Figure 10: Plots for stable regular ABG wormholes in the context of MGCG with $\xi_{0}=\gamma=1$ and different values of $Q$. We plot throat radius $a_{0}$ and parameter $w$ along abscissa and ordinate, respectively, where $a_{0}=r$ at thinshell. 

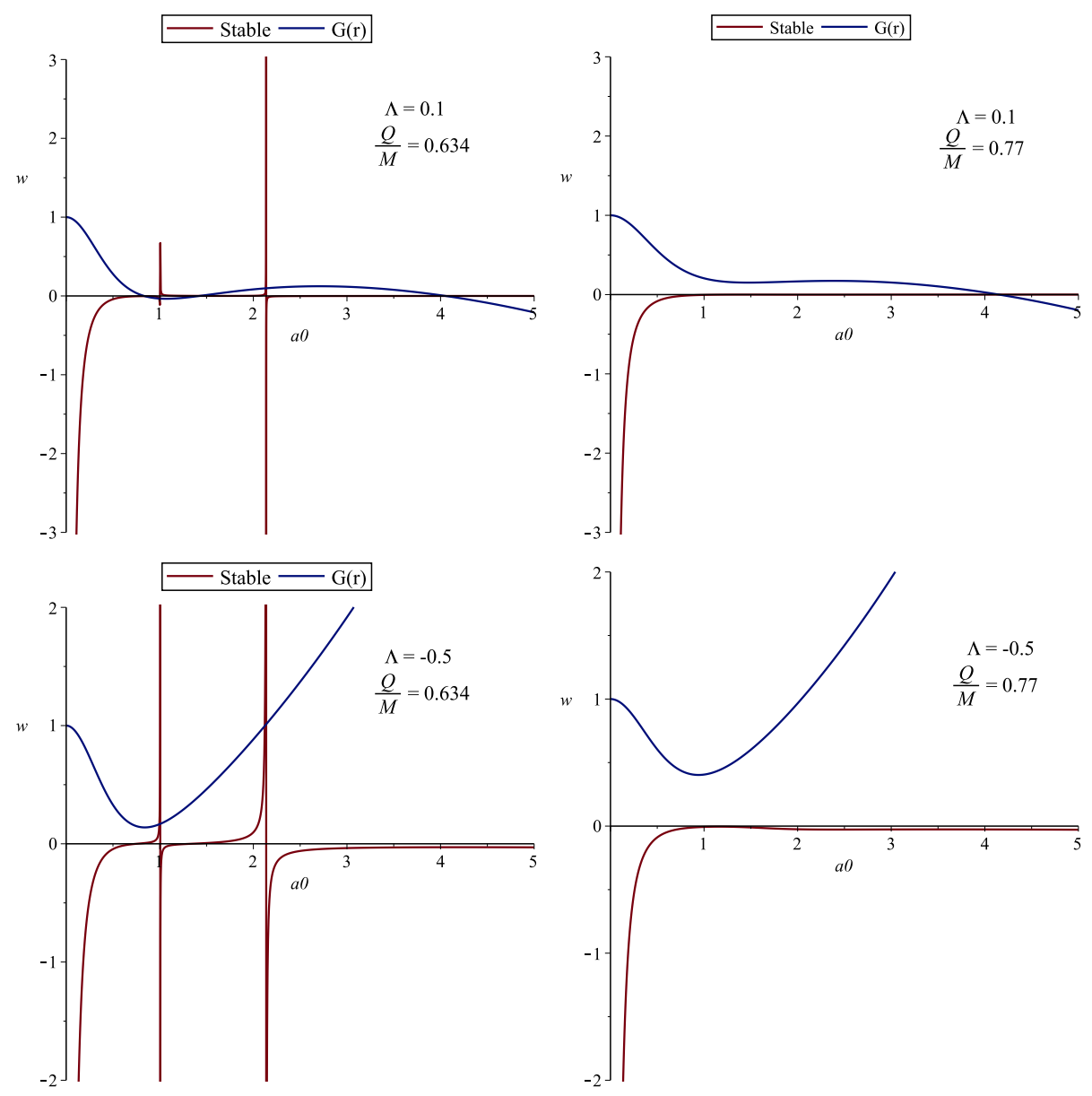

Figure 11: Plots for stable regular ABG wormhole solutions with MGCG, $\xi_{0}=\gamma=1$ and $\Lambda=0.1,-0.5$. 
that more stable wormhole solutions exist for $\frac{Q}{M}=0.634$ and stability area decreases by increasing $\frac{Q}{M}$. We find only one stable region for larger values of $\frac{Q}{M}$. It is observed that more stable regions exist for de Sitter and antide Sitter spacetimes in comparison to the spacetime without $\Lambda$ (Figure 13). We obtain maximum stable regions for $\frac{Q}{M}=0.634$ in de Sitter and antide Sitter spacetimes. We investigate that the effect of logarithmic gas with inclusion of $\Lambda$ is to enhance the stability regions for regular ABG wormhole configurations as depicted in our numerical plots.

\section{Stability Analysis under Velocity Pertur- bations}

Now, we study stability of regular ABG thin-shell wormholes against velocity perturbations. We take small velocity perturbations about static configuration $a=a_{0}$ such that we can consider an approximately static fluid for exotic matter after any perturbation. This fact leads to an assumption that one can take dynamic EoS for wormhole same as that of static EoS [12]. In this context, we use Eq.(14) which leads to

$$
p=-\frac{1}{2}\left(1+\frac{a G^{\prime}(a)}{2 G(a)}\right) \sigma .
$$

Inserting $\sigma$ and $p$ from Eqs.(8) and (9), it follows that

$$
\ddot{a}-\frac{G^{\prime}(a)}{2 G(a)} \dot{a}=0
$$

which corresponds to the wormhole's throat motion in one-dimension. Integration of this equation yields

$$
\dot{a}=\dot{a}_{0} \frac{\sqrt{G(a)}}{\sqrt{G\left(a_{0}\right)}},
$$

whose second integration gives

$$
\frac{\dot{a_{0}}}{\sqrt{G\left(a_{0}\right)}}\left(\tau-\tau_{0}\right)=\int_{a_{0}}^{a} \frac{d a}{\sqrt{G(a)}} .
$$

It is mentioned here that $\dot{a}_{0}$ is assumed to be non-zero initial small velocity

of throat. In the following, we provide examples to analyze stability under small velocity perturbations. 

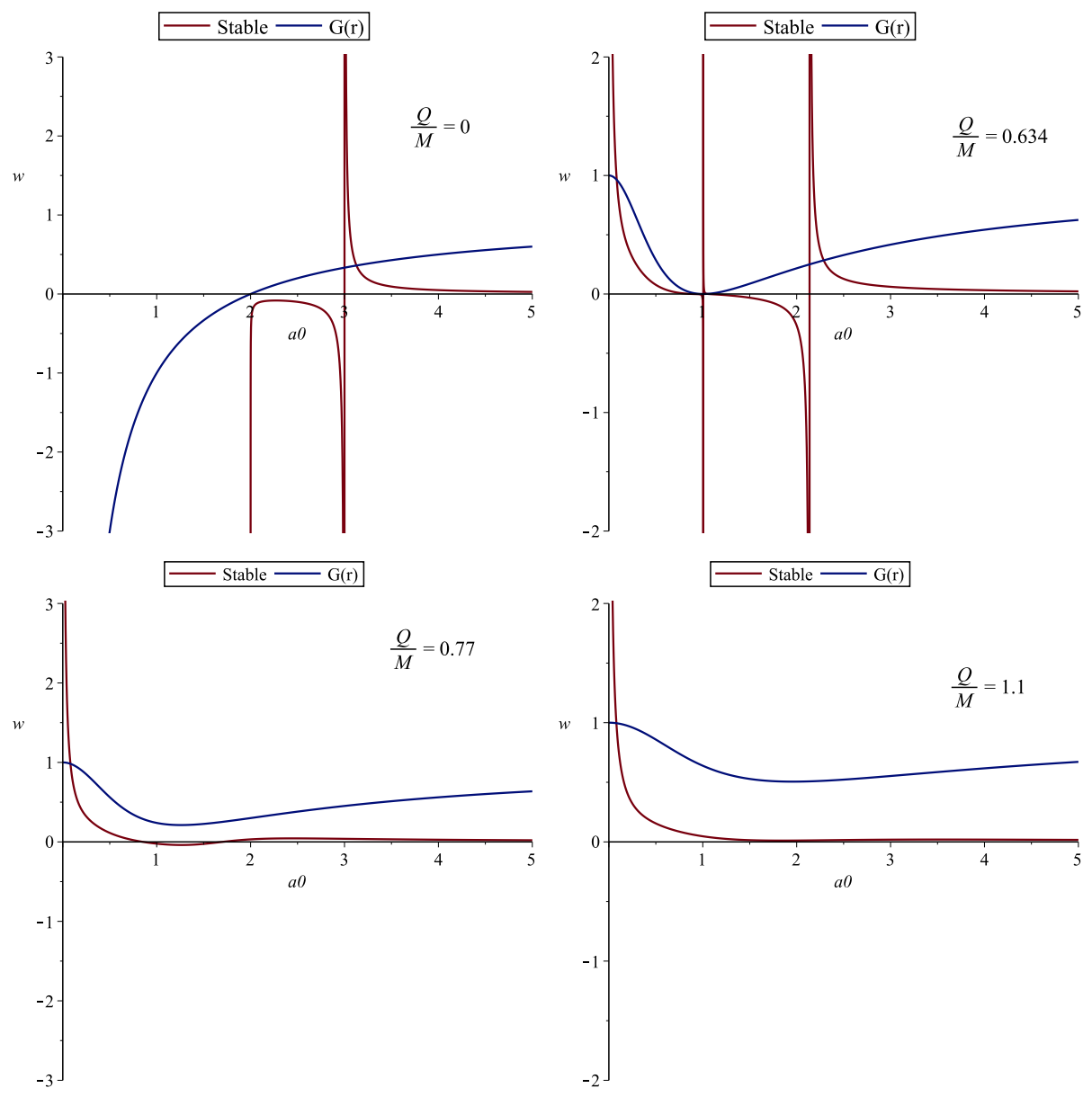

Figure 12: Plots for logarithmic gas EoS with $\frac{Q}{M}=0,0.634,0.77,1.1$. We plot throat radius $a_{0}$ and parameter $w$ along abscissa and ordinate, respectively, where $a_{0}=r$ at thin-shell. 

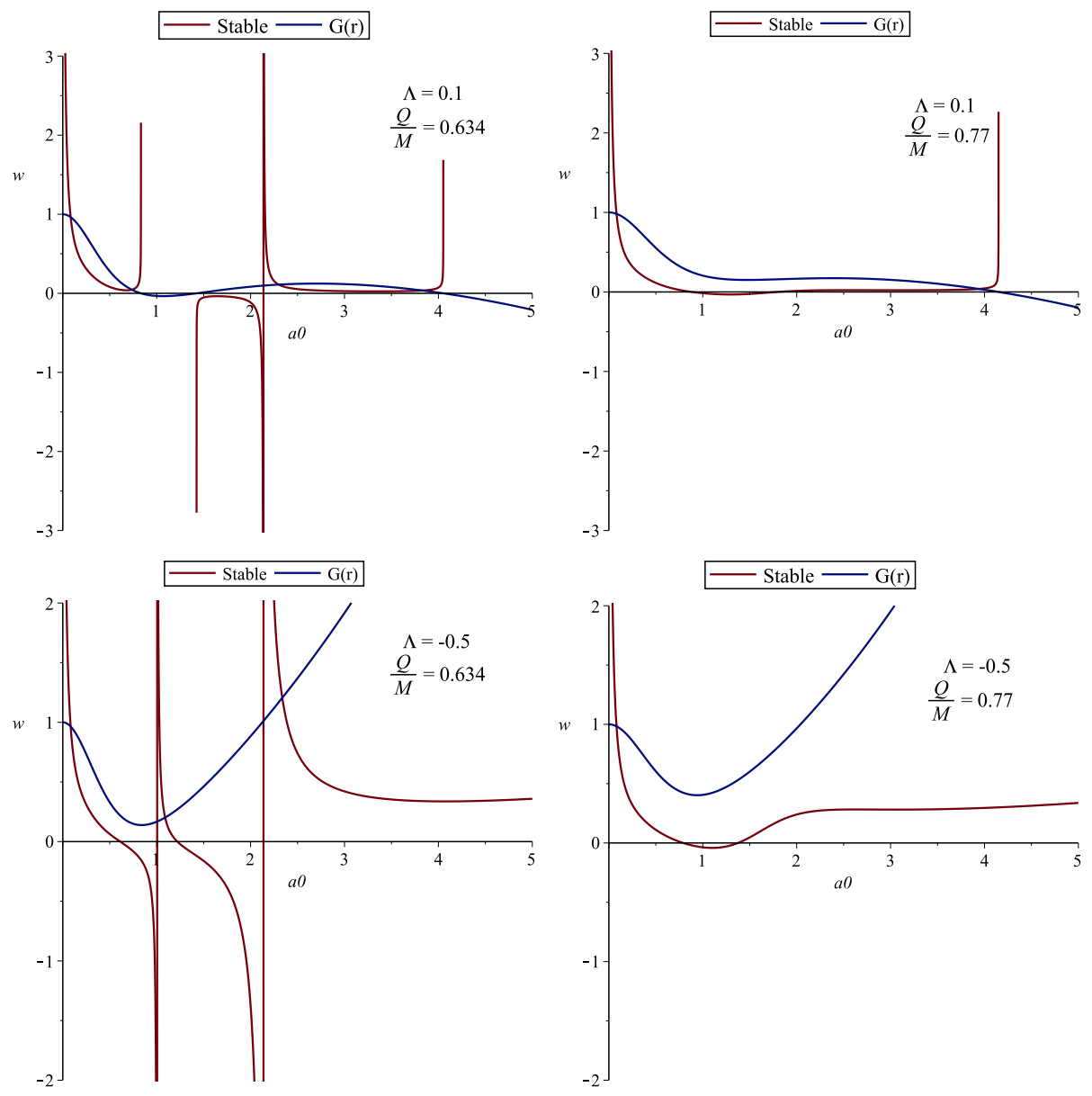

Figure 13: Plots for stable regular ABG wormholes with logarithmic gas EoS and $\Lambda=0.1,-0.5$. 


\subsection{The Schwarzschild Case}

For Schwarzschild metric function $G(a)=1-\frac{2 M}{a}$, Eq.(31) yields

$$
\frac{\dot{a_{0}}}{\sqrt{G\left(a_{0}\right)}}\left(\tau-\tau_{0}\right)=a \sqrt{G(a)}-a_{0} \sqrt{G\left(a_{0}\right)}+M \ln \left(\frac{a-M+a \sqrt{G(a)}}{a_{0}-M+a_{0} \sqrt{G\left(a_{0}\right)}}\right)
$$

which shows the non-oscillatory motion. Thus, the wormhole throat for Schwarzschild case will be unstable under small velocity perturbations.

\subsection{The ABG Case}

For the regular ABG BH, Eq. (31) upto the third order of $Q$ gives

$$
\begin{aligned}
\frac{\dot{a_{0}}}{\sqrt{G\left(a_{0}\right)}}\left(\tau-\tau_{0}\right) & =a \sqrt{G(a)}-a_{0} \sqrt{G\left(a_{0}\right)}+M \ln \left(\frac{a-M+a \sqrt{G(a)}}{a_{0}-M+a_{0} \sqrt{G\left(a_{0}\right)}}\right) \\
& -\frac{\left(a-a_{0}\right)^{3}}{6}\left(\frac{1}{Q^{2}}-\frac{2 M}{Q^{3}}\right),
\end{aligned}
$$

which again indicates the non-oscillating motion. Consequently, the wormhole throat for regular ABG spacetime will remain unstable under velocity perturbations. Also, Eq.(29) provides the throat's acceleration $\ddot{a}=\frac{G^{\prime}(a)}{2 G(a)} \dot{a}$ to be positive leading to unstable ABG wormhole configurations.

\section{Conclusions}

This paper is devoted to construct regular ABG thin-shell wormholes by implementing the Visser's cut and paste technique. We have formulated the surface stresses by using Lanczos equation which indicate the existence of exotic matter due to the violation of NEC and WEC. It is found that the wormhole has attractive or repulsive characteristics for $a^{r}>0$ and $a^{r}<0$, respectively. Moreover, we have quantified the total amount of exotic matter by the volume integral theorem which shows that a small quantity of exotic matter is required to support the wormhole. We have analyzed stability of the constructed thin-shell wormholes by incorporating the effects of increasing values of electric charge. We have taken $a>r_{h}$ in order to neglect the presence of event horizons for wormhole's viability. 
We have considered perturbation of the form $p=\Phi(\sigma)$ and studied stability conditions graphically for $\Delta^{\prime \prime}>0$. To find any realistic dark energy candidate, we have chosen linear, logarithmic, CG, GCG and MGCG models as exotic matter at the wormhole throat. The trivial case $\frac{Q}{M}=0$ corresponds to Schwarzschild spacetime. We have investigated stable regions within a physically acceptable range of different parameters (Figures 4-13). We have also plotted the function $G(r)$ to evaluate the position of wormhole throat and event horizon of $\mathrm{BH}$, where we have choosen $a_{0}=r$ at thin-shell (hypersurface $\Sigma$ ). It is observed that the stability areas for linear gas tend to decrease by increasing the values of $\frac{Q}{M}$ while CG shows the least stable regions for the respective wormhole solutions. For GCG and MGCG, we have analyzed more stability regions which decrease gradually with increasing $\frac{Q}{M}$ and diminish to only one stable region for $\frac{Q}{M}=1.1$. It is found that the role of logarithmic gas is to enhance stable regions for regular ABG wormhole configurations. We have concluded that the wormhole stability highly depends on the values of electric charge.

We have also investigated the stable regions for regular ABG thin-shell wormholes in de Sitter $(\Lambda=0.1)$ and anti-de Sitter $(\Lambda=-0.5)$ backgrounds. In this context, linear gas shows more stable regions as compared to the regions for anti-de Sitter spacetime. It is observed that GCG gives maximum stability areas for regular ABG thin-shell wormholes corresponding to different values of $\frac{Q}{M}$ with $\Lambda<0$. For MGCG and logarithmic gas, it is found that the increasing value of $\frac{Q}{M}$ shows enlargement in the stable regions and maximum stable regions are obtained for $\Lambda=-0.5$. We have also discussed small velocity dependent perturbations and analyzed that the constructed wormhole configurations will be unstable under these perturbations. A consequence of this instability is that the wormhole throat may expand or collapse to a $\mathrm{BH}$ depending on the signs of velocity. It is observed that there are more stable wormhole solutions in de Sitter and anti-de Sitter spacetimes in comparison to the case without $\Lambda$. We conclude that stability regions may expand/shrink by tuning the values of charge and other parameters.

\section{References}

[1] Hayward, S.A.: arXiv gr-qc/0203051.

[2] Einstein, A. and Rosen, N.: Phys. Rev. D 48(1935)73. 
[3] Morris, M. and Thorne, K.: Am. J. Phys. 56(1988)395.

[4] Israel, W.: Nuovo Cimento B 44S10(1966)1; Erratum B48(1967)463.

[5] Nandi, K.K., Zhang, Y.Z. and Kumar, K.B.V.: Phys. Rev. D 70(2004)127503.

[6] Visser, M.: Phys. Rev. D 39(1989)3182; Lorentzian Wormholes (AIP Press, 1996).

[7] Bardeen, J.M.: Non-singular General Relativistic Gravitational Collapse (Proc. International Conference GR5, Tbilisi, USSR, 1968).

[8] Ayon-Beato, E. and Garcia, A.: Phys. Rev. Lett. 80(1998)5056.

[9] Hayward, S.A.: Phys. Rev. Lett. 96(2006)031103.

[10] Halilsoy, M., Ovgun, A. and Mazharimousavi, S.H.: Eur. Phys. J. C $\mathbf{7 4}(2014) 2796$.

[11] Kim, S.W. and Lee, H.: Phys. Rev. D 63(2001)064014.

[12] Sharif, M. and Azam, M.: Eur. Phys. J. C 73(2013)2554.

[13] Sharif, M. and Mumtaz, S.: Astrophys. Space Sci. 352(2014)729.

[14] Sharif, M. and Mumtaz, S.: Can. J. Phys. 93(2015)12.

[15] Lobo, F.S.N. et al.: arXiv:1512.08474.

[16] Lobo, F.S.N.: Phys. Rev. D 71(2005)124022.

[17] Capozziello, S. et al.: J. Cosmol. Astropart. Phys. 04(2005)005.

[18] Eiroa, E.F. and Simeone, C.: Phys. Rev. D 76(2007)024021.

[19] Eiroa, E.F.: Phys. Rev. D 80(2009)044033.

[20] Sharif, M. and Azam, M.: J. Cosmol. Astropart. Phys. 05(2013)025.

[21] Sharif, M. and Mumtaz, S.: Adv. High Energy Phys. 2014(2014)639759.

[22] Sharif, M. and Mumtaz, S.: Adv. High Energy Phys. 2016(2016)2868750. 
[23] Mazharimousavi, S.H. and Halilsoy, M.: Eur. Phys. J. C 74(2014)3073.

[24] Hawking, S. W. and Ellis, G. F. R.: The Large Scale Structure of Spacetime (Cambridge University Press, 1975).

[25] Stuchlík, Z. and Schee, J.: Int. J. Mod. Phys. D 24(2015)1550020.

[26] Schee, J. and Stuchlík, Z.: J. Cosmol. Astropart. Phys. 06(2015)048.

[27] Wen-Juan, M., Rong-Gen, C. and Ru-Keng, S.: Commun. Theor. Phys. 46(2006)453.

[28] Stuchlík, Z. and Hledík, S.: Phys. Rev. D 60(1999)044006.

[29] Stuchlík, Z.: Mod. Phys. Lett. A 20(2005)561.

[30] Faraoni, V.: Physics of the Dark Universe 11(2016)11.

[31] Fernando, S.: Int. J. Mod. Phys. D 24(2015)1550104.

[32] Lobo, F.S.N: Class. Quantum Grav. 21(2004)4811.

[33] Sharif, M. and Mumtaz, S.: Astrophys. Space Sci. 361(2016)218.

[34] Richarte, M.G. and Simeone, C.: Phys. Rev. D 80(2009)104033. 